\title{
Research Paper: The Validity and Reliability of the Persian Version of the Leeds Dependence Questionnaire
}

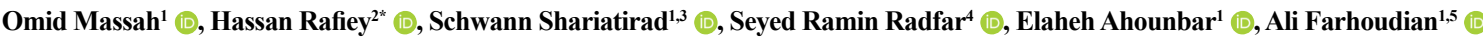 \\ 1. Substance Abuse and Dependence Research Center, University of Social Welfare and Rehabilitation Sciences, Tehran, Iran. \\ 2. Social Welfare Management Research Center, University of Social Welfare and Rehabilitation Sciences, Tehran, Iran. \\ 3. Students Research Center of International Campus, Tehran University of Medical Sciences, Tehran, Iran. \\ 4. UCLA Integrated Substance Abuse Programs, University of California, Los Angeles, California, United States \\ 5. Department of Psychiatry, School of Medicine, Tehran University of Medical Sciences, Tehran, Iran.
}

\begin{tabular}{l|l}
\hline $\begin{array}{c}\text { Use your device to scan } \\
\text { and read the article online }\end{array}$ \\
sion of the Leeds Dependence Questionnaire. Iranian Rehabilitation Journal. 2019; 17(2):91-96. http://dx.doi.org/10.32598/irj.17.2.91 \\
dol http://dx.doi.org/10.32598/irj.17.2.91
\end{tabular}

Article info:

Received: 10 Dec 2018

Accepted: 25 Feb 2019

Available Online: 01 June 2019

Keywords:

Leeds dependence questionnaire, Substancerelated disorders, Iran

\section{A B S T RACT}

Objectives: The follow-up treatment or rehabilitation and monitoring of patients with drug abuse disorders require an efficient tool with a convenient and short-time implementation to measure psychological aspects of dependence. The Leeds Dependence Questionnaire (LDQ) has such features; thus, the present study aimed at evaluating the validity and reliability of its Persian version.

Methods: The current validation study explored the data obtained from 142 substance dependent patients selected from drug addiction treatment centers in Tehran City, Iran. The criterion-related validity of the Persian version of the LDQ was evaluated by measuring its correlation with the Substance Dependence Severity Scale (SDSS). The construct validity of the Persian version of the LDQ was evaluated using the General Health Questionnaire (GHQ). Moreover, the internal consistency and reliability of this scale were calculated by Cronbach's alpha coefficient and test-retest reliability methods, respectively.

Results: The correlation coefficients between the LDQ and SDSS and GHQ were 0.773 and 0.780 , respectively; the correlations were statistically significant $(\mathrm{P}<0.001)$. A Cronbach's alpha coefficient of 0.809 was obtained by investigating the internal consistency of the tool. Test-retest reliability for a two-week interval was 0.963 .

Discussion: The criterion-related validity, construct validity, internal consistency, and testretest reliability values of the Persian version of the LDQ are approvable; therefore, it can be applied as a valid and reliable tool on the Iranian population.

\section{* Corresponding Author:}

Hassan Rafiey, MD.

Address: Social Welfare Management Research Center, University of Social Welfare and Rehabilitation Sciences, Tehran, Iran.

Tel: +98 (21) 71732863

E-mail: hassan441015@gmail.com 


\section{Highlights}

- The Persian version of the LDQ has an appropriate criterion and construct validity.

- The Persian version of LDQ has appropriate internal consistency and test-retest reliability.

- The Persian version of LDQ is an appropriate and reliable tool to assess the severity and type of substance dependence.

\section{Plain Language Summary}

To evaluate the effectiveness and success rate of substance dependence treatment and rehabilitation programs, appropriate tools developed based on the culture, language, and circumstances of the population are required. The present research reported the successful validation of an applicable tool that measures substance dependence: The Persian version of the Leeds Dependence Questionnaire (LDQ).

\section{Introduction}

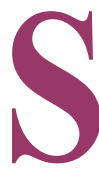

ubstance dependence is a bio-psychosocial problem; investigating substance dependence falls beyond the type, amount, and frequency of abuse [1]. Significant advances are made in the treatment of drug abuse disorders over the past two decades. Moreover, various evidence-based therapeutic approaches are well developed, which the efficacy and cost-effectiveness of each are measured. The continuance of such process and the better and more accurate follow-up of the treatment trend are subject to a valid and reliable examination of the signs and symptoms of progression or decline in disease. Obviously, any evaluation requires an appropriate and valid measurement tool, tailored to specific circumstances of each country $[2,3]$.

Application of a comprehensive substance dependence treatment and rehabilitation program requires a precise diagnosis of drug abuse disorder and assessment of its severity. The accurate recognition of such conditions and evaluation of the effectiveness of provided services at each stage of a comprehensive treatment plan are important factors in an appropriate proceeding. Diagnosis of a drug abuse disorder, and more importantly, determining its severity are often difficult [4]. Substance dependence severity cannot be measured via determining the type, amount, and duration of abuse [5].

Leeds Dependence Questionnaire (LDQ) is a practical and useful tool to assess substance dependence severity, applicable to alcohol, opiates, and psychostimulants [6]. Raistrick believed that the special role of a therapist is to treat "dependency". On this basis, the LDQ was developed as part of a comprehensive treatment package to measure one component: dependency and dependence treatment [7]. The LDQ is mainly focused on the psychological aspects of substance dependence and measures the same criteria as considered in ICD-11, which are, of course, very similar to DSM-V (the Diagnostic and Statistical Manual of Mental Disorders, fifth edition).

The LDQ is emphasized on the importance of extremely increasing the substance impact on the abusers, their priority in obtaining and maintaining the desired impact of substance, and the tolerance phenomenon, instead of pointing out withdrawal symptoms, as the main symptoms of substance dependence (because of less important of withdrawal symptoms). As a result, the LDQ automatically excludes patients receiving opiates due to surgery, pain, or neurological complications, but have no willing to consume those [6].

The LDQ is a 10-item self-report scale, designed in accordance with the 10 obvious symptoms of substance dependence. A question is designed for evaluating each symptom, which is answered on a four-point Likert scale from zero to three, and the total score of 30 .

The 10 symptoms were considered in the LDQ, and the main contents of the related questions, are briefly mentioned as follows: 1. Preoccupation with substance: thinking about the next time of the use; 2 . The salience of substance use: this is considered in comparison with other daily living activities; 3 . The compulsion to start: the lack of control over not using; 4. Planning for substance use: a daily schedule based on obtaining and using substances; 5. Maximize effect: Consuming the substance in a route that maximizes its effects; 6 . 
Narrowing of using repertoire: very frequent use, and repeated use; 7 . The compulsion to continue: the lack of control over the discontinuation of each turn; 8 . The primacy of effect: the priority of substance effect and not the type of substance (e.g. any kind of stimulating agent for $\mu$ receptor); 9. Constant state: attempting to reuse as soon as the substance effect decreases slightly; 10. Cognitive set: solving life problems or coping with them, without using substance [6].

There was a need for a scale to measure the severity of substance dependence with a convenient and short-time implementation. Moreover, it had to appropriately evaluate obvious substance dependence symptoms. Thus, the present study aimed at providing the Persian version of LDQ and evaluating its validity and reliability.

\section{Methods}

The present study aimed at determining the validity of the Persian version of LDQ. The questionnaire was translated into Persian after obtaining permission from the developer. Then, the tool was back-translated into English and sent to the developer for examining the content validity of the items.

The developer's comments were considered and the tool was revised based on the changes. Then the edited version was resent to the developer for the final approval. In the next step, the Persian version of the questionnaire, as well as the Substance Dependence Severity Scale (SDSS) were performed to examine the criterion-related validity of the tool on a group of drug dependent patients started treatment for drug use disorders in the relevant settings in Tehran City, Iran. Furthermore, the General Health Questionnaire (GHQ28) was conducted on the same sample population to explore the construct validity of the tool via correlation with LDQ. The Persian version of LDQ was completed by a number of study participants to evaluate its testretest reliability, after two weeks.

The statistical population of the present research consisted of all patients with drug abuse disorders in 2017 in Iran. The study samples were selected using the convenient sampling method from those referring to the outpatient drug use treatment centers in Tehran. The sample size was 120 using the related formula . Considering the probability dropouts, as well as the lack of access to all the subjest at the retest phase, the final sample size was considered 150 . The study participants were selected by simple convenient sampling method from three clinics; eventually, the obtained data from 142 subjects were analyzed.
The construct validity, criterion-related validity, and the reliability of the main version of the LDQ were measured and approved by Severity of Alcohol Dependence Questionnaire (SADQ), Severity of Opiates Dependence Questionnaire (SODQ), GHQ, and Social Function Auestionnaire (SFQ); the same were endorsed by subsequent studies as well [6, 8-10].

The reported test-retest reliability of the SDSS varied from good to excellent (intra-class correlation coefficient: 0.75-0.88) [11-13]. The Cronbach's alpha coefficient and the test-retest reliability of the Persian version of SDSS was reported 0.77 and 0.97 , respectively [14].

The concurrent validity of the 28-item GHQ and its subscales ranged 0.83 to 0.95 in respect to construct and criterion validity through various studies conducted by the World Health Organization [15]. The validity of the Persian version of GHQ was evaluated by the three methods of test-rest reliability, split half, and Cronbach's $\alpha$ and the results were $0.70,0.93$, and 0.90 , respectively [16]. In addition, its Cronbach's $\alpha$ coefficient was 0.69 for all aspects, by another research [17]. The obtained data were analyzed with SPSS V. 23. In addition to descriptive statistics, Pearson's correlation coefficient was applied to examine the validity of the assumptions.

\section{Results}

The Mean \pm SD of study participants' age was $39.49 \pm 10.78$ years; ranged 19 to 72 . Moreover, 131 $(92.3 \%)$ subjects were male and $11(7.7 \%)$ female.

The main used substances by the study participants were as follows: opium $(n=47)$, heroin $(n=36)$, shireh (a refined opium extract $)(n=20)$, crystal methamphetamine $(n=12)$, illegal methadone $(n=10)$, alcohol $(n=6)$, and other substance types were abused by the rest of the subjects. In total, 72 patients were polydrug users. The second most frequently used substance was methamphetamine (crystal methamphetamine), where 37 patients reported its consumption as their second choice. The correlation between the scores of LDQ and SDSS was used to assess the criterion-related validity of the Persian version of LDQ.

According to Table 1, the criterion validity of the Persian version of LDQ was acceptable. The correlation between the scores of LDQ and GHQ were explored to evaluate the construct validity of the Persian version of LDQ. According to Table 2, the construct validity of the Persian version of the LDQ was acceptable. 
Table 1. Pearson's correlation coefficient for LDQ and SDSS scores

\begin{tabular}{cccc}
\hline Variable & Mean \pm SD & Pearson's Correlation Coefficient & P \\
\hline The total score of LDQ & $22.52 \pm 5.30$ & & $<.773$ \\
The total score of SDSS & $32.41 \pm 5.04$ & & $<001$ \\
\hline
\end{tabular}

Iranian Rehabilitation \ourna

Table 2. Pearson's correlation coefficient for LDQ and GHQ scores

\begin{tabular}{cccc}
\hline Variable & Mean \pm SD & Pearson's Correlation Coefficient & P \\
\hline The total score of LDQ & $22.52 \pm 5.30$ & 0.780 & $<0.001$ \\
The total score of GHQ & $60.99 \pm 10.73$ & & \\
\hline
\end{tabular}

Iranian Rehabilitation Journa

Cronbach's alpha coefficient was used to assess the internal consistency of the Persian version of LDQ; the Cronbach's alpha coefficient was 0.809 , which indicated the high internal consistency of the scale. Furthermore, to evaluate the test-retest reliability of the Persian version of LDQ, the obtained data from 36 participants at two different time points (a two-week interval) were evaluated. The relevant findings are presented in Table 3. In addition, the intraclass correlation coefficient was applied to measure the test-retest reliability; the obtained data are shown in Table 4.

\section{Discussion}

The current study evaluated the validity and reliability of the Persian version of LDQ. Evaluation of the criterion validity of the Persian version of LDQ suggested a correlation coefficient of 0.773 , which was statistically significant. Raistrick in a study reporting the history of this scale assessed its concurrent criterion validity and reported its correlation with SODQ and SADQ as good. Different investigations reported various correlation coefficients from 0.69 to 0.80 , which were statistically significant at $\mathrm{P}<0.001$ [6]. The correlation coefficient of the criterion validity of this tool indicated its good validity to evaluate substance dependence.

Investigating the construct validity of the Persian version of the LDQ suggested a correlation coefficient of 0.780 , which was statistically significant; it indicates the consistence between the scores of the Persian version of LDQ and the Persian version of GHQ. In addition, Raistrick provided a comprehensive report on the development of LDQ, in which the correlation between the scores of LDQ and GHQ was examined in two groups of patients; they reported the correlation coefficient of 0.56

Table 3. Pearson's correlation coefficient for test-retest scores of LDQ

\begin{tabular}{cccc}
\hline Variable & Mean \pm SD & Pearson's Correlation Coefficient & P \\
\hline The total score of LDQ (test) & $23.44 \pm 4.74$ & & $<.963$ \\
The total score of LDQ (retest) & $22.19 \pm 4.26$ & & $<0.001$ \\
\hline
\end{tabular}

Table 4. The intraclass correlation coefficient for test-retest scores of LDQ

\begin{tabular}{|c|c|c|c|c|c|c|c|}
\hline \multirow{2}{*}{ Variable } & \multirow{2}{*}{$\begin{array}{l}\text { Intraclass } \\
\text { Correlation }\end{array}$} & \multicolumn{2}{|c|}{ 95\% Confidence Interval } & \multicolumn{4}{|c|}{ F Test With True Value 0} \\
\hline & & Lower Bound & Upper Bound & Value & $\mathrm{df}_{1}$ & $\mathrm{df}_{2}$ & Sig. \\
\hline Single measures & 0.957 & 0.918 & 0.978 & 45.964 & 35 & 35 & 0.000 \\
\hline Average measures & 0.978 & 0.957 & 0.989 & 45.964 & 35 & 35 & 0.000 \\
\hline
\end{tabular}


and 0.70 , which were statistically significant at $\mathrm{P}<0.01$ and $<0.001$, respectively [6]. Another research explored the construct validity between the scores of LDQ and GHQ and reported the correlation coefficient of 0.50 that was statistically significant at $\mathrm{P}<0.001$ [8]. The convergent construct validity between the LDQ and the brief symptom inventory-18 was explored by another study where the correlation coefficient ranged 0.44 to 0.51 that was statistically significant at $\mathrm{P}<0.001$ [9].

The internal consistency of LDQ was examined in the current study by Cronbach's alpha that was 0.809 , reflecting the high internal consistency between the items of the scale. The Cronbach's alpha coefficients reported in previous studies were similar to that obtained in the present study. For instance, Lennings (1999) in a study reported the internal consistency of LDQ from two studies; the Cronbach's alpha coefficients were 0.89 and 0.89 [10]. Raistrick, the LDQ developer, reported Cronbach's alpha coefficients of 0.94 and 0.90 for LDQ from two studies $[6,8]$. The internal consistency of LDQ was reported equal to Cronbach's alpha coefficient, 0.93 , in another investigation [9].

The current study evaluated the correlation between the data obtained from two stages of the LDQ implementation with a two-week interval on 36 patients to calculate the test-retest reliability of the scale. The relevant data were achieved using Pearson correlation coefficient that was 0.963 . The initial investigation of Raistrick suggested the test-retest reliability of 0.95 [6]. Another study explored the test-retest reliability of LDQ by reimplementing the questionnaire on 25 subjects within a two-week interval; they reported a test-retest correlation coefficient of 0.85 [10].

\section{Conclusion}

The Persian version of LDQ is a very reliable and valid tool for examining the severity of dependence on different substances, and considering its feasibility, LDQ can be well applied to evaluate substance dependence and its severity by the treatment programs.

\section{Ethical Considerations}

\section{Compliance with ethical guidelines}

This study was approved by the Ethics Committee of University of Social Welfare and Rehabilitation Sciences (code: IR.USWR.REC.1395.329).

\section{Funding}

This article was part of a research project (code: 95/1024) and was funded by the Research Deputy of University of Social Welfare and Rehabilitation Sciences.

\section{Authors' contributions}

Study design: Hassan Rafiey; Literature search, review, translation of the questionnaire, corresponding: Omid Massah, Schwann Shariatirad; Conduction of the study, data collection: Omid Massah, Elaheh Ahounbar; Data entry and analysis: Omid Massah, Elaheh Ahounbar, Ali Farhoudian, Ramin Radfar; Writing the manuscript: Omid Massah; and Writing, reading, editing, and approving the final version of the manuscript: All authors.

\section{Conflict of interest}

The authors declared no conflict of interest.

\section{Acknowledgments}

The authors acknowledge their gratitude to the colleagues and participants from different treatment centers who contributed to this research. In addition, the authors sincerely appreciate Mr. Duncan Raistrick for approving to develop the Persian version of the questionnaire, as well as his contribution and guidance on the research.

\section{References}

[1] Walters GD. The addiction concept: Working hypothesis or self-fulfilling prophesy. New Jersey: Prentice Hall; 1999.

[2] McLellan AT, Hagan TA, Levine M, Meyers K, Gould F, Bencivengo $\mathrm{M}$, et al. Does clinical case management improve outpatient addiction treatment. Drug and Alcohol Dependence. 1999; 55(1-2):91-103. [DOI:10.1016/S0376-8716(98)00183-5]

[3] Glasner-Edwards S, Rawson R. Evidence-based practices in addiction treatment: Review and recommendations for public policy. Health Policy. 2010; 97(2-3):93-104. [DOI:10.1016/j. healthpol.2010.05.013] [PMID] [PMCID]

[4] Leshner AI. Science-based views of drug addiction and its treatment. JAMA. 1999; 282(14):1314-6. [DOI:10.1001/ jama.282.14.1314] [PMID]

[5] McLellan AT, Luborsky L, Woody GE, O'Brien CP. An improved diagnostic evaluation instrument for substance abuse patients: The AddictionSeverity Index. Journal of Nervous and Mental Disease. 1980; 168(1):26-33. [DOI:10.1097/00005053198001000-00006] [PMID] 
[6] Duncan R, Jackie B, Gillian T, Jeremy W, Jeff A, Carolun H. Development of the Leeds Dependence Questionnaire (LDQ): A questionnaire to measure alcohol and opiate dependence in the context of a treatment evaluation package. Addiction. 1994; 89(5):563-72. [DOI:10.1111/j.1360-0443.1994.tb03332.x]

[7] Duncan R. Substance Problems: the future of specialist services. British Journal of Addiction. 1988; 83(4):349-50. [DOI:10.1111/j.1360-0443.1988.tb00480.x]

[8] Heather N, Raistrick D, Tober G, Godfrey C, Parrott S. Leeds dependence questionnaire: New data from a large sample of clinic attenders. Addiction Research \& Theory. 2001; 9(3):25369. [DOI:10.3109/16066350109141753]

[9] Kelly JF, Magill M, Slaymaker V, Kahler C. Psychometric validation of the Leeds Dependence Questionnaire (LDQ) in a young adult clinical sample. Addictive Behaviors. 2010; 35(4):331-6. [DOI:10.1016/j.addbeh.2009.11.005] [PMID] [PMCID]

[10] Lennings CJ. An evaluation of the Leeds dependence questionnaire. Journal of Child \& Adolescent Substance Abuse. 1999; 8(3):73-87. [DOI:10.1300/J029v08n03_05]

[11] Miele GM, Carpenter KM, Cockerham MS, Trautman KD, Blaine J, Hasin DS. Substance Dependence Severity Scale (SDSS): Reliability and validity of a clinician-administered interview for DSM-IV substance use disorders. Drug and Alcohol Dependence. 2000; 59(1):63-75. [DOI:10.1016/S03768716(99)00111-8]

[12] Miele GM, Carpenter KM, Cockerham MS, Trautman KD, Blaine J, Hasin DS. Substance dependence severity scale: Reliability and validity for ICD-10 substance use disorders. Addictive Behaviors. 2001; 26(4):603-12. [DOI:10.1016/S03064603(00)00137-4]

[13] Miele GM, Carpenter KM, Cockerham MS, Trautman KD, Blaine J, Hasin DS. Concurrent and predictive validity of the Substance Dependence Severity Scale (SDSS). Drug and Alcohol Dependence. 2000; 59(1):77-88. [DOI:10.1016/S03768716(99)00110-6]

[14] Motlaq ME, Garrusi B, Shamsinejad B. Reliability and validity of a Persian version of Substance Dependence Severity Scale (SDSS). Addiction \& Health. 2009; 1(2):87-91. [PMID] [PMCID]

[15] Goldberg DP, Gater R, Sartorius N, Ustun TB, Piccinelli $\mathrm{M}$, Gureje $\mathrm{O}$, et al. The validity of two versions of the GHQ in the WHO study of mental illness in general health care. Psychological Medicine. 1997; 27(1):191-7. [DOI:10.1017/ S0033291796004242] [PMID]

[16] Taghavi S. [Validity and reliability of Persian version of general health questionnaire (Persian)]. Journal of Psychology. 2002; 5(4):381-98.

[17] Valizadeh R, Kavari zadeh F, Yasemi M, Yasemi MR, Rashidbeigi $M$, Tavan $H$. Psychometric properties of the Persian version of General Health Questionnaire (GHQ -28). Journal of Ilam University of Medical Sciences. 2015; 22(6):44-50. 